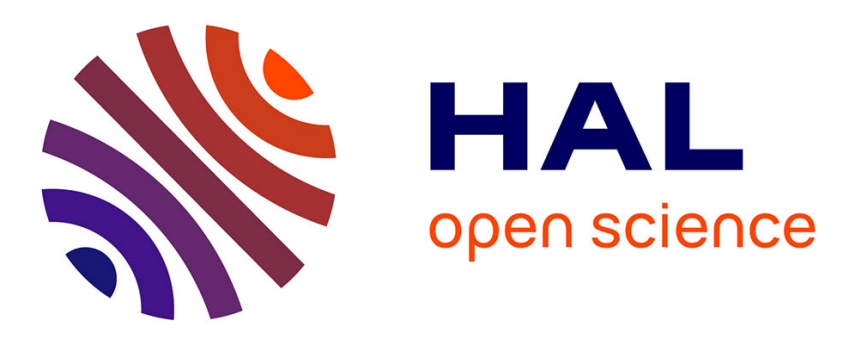

\title{
The mechanical resonances of electrostatically coupled nanocantilevers
}

\author{
S. Perisanu, Thomas Barois, P. Poncharal, T. Gaillard, A. Ayari, S. T. \\ Purcell, P. Vincent
}

\section{- To cite this version:}

S. Perisanu, Thomas Barois, P. Poncharal, T. Gaillard, A. Ayari, et al.. The mechanical resonances of electrostatically coupled nanocantilevers. Applied Physics Letters, 2011, 98 (6), pp.063110. 10.1063/1.3553779 . hal-01565121

\section{HAL Id: hal-01565121 \\ https://hal.science/hal-01565121}

Submitted on 29 Sep 2017

HAL is a multi-disciplinary open access archive for the deposit and dissemination of scientific research documents, whether they are published or not. The documents may come from teaching and research institutions in France or abroad, or from public or private research centers.
L'archive ouverte pluridisciplinaire HAL, est destinée au dépôt et à la diffusion de documents scientifiques de niveau recherche, publiés ou non, émanant des établissements d'enseignement et de recherche français ou étrangers, des laboratoires publics ou privés. 


\title{
The mechanical resonances of electrostatically coupled nanocantilevers
}

\author{
S. Perisanu, a) T. Barois, P. Poncharal, T. Gaillard, A. Ayari, S. T. Purcell, and P. Vincent ${ }^{\text {b) }}$ \\ LPMCN, Université Claude Bernard Lyon 1 et CNRS, UMR 5586, F-69622 Villeurbanne, France
}

(Received 8 December 2010; accepted 20 January 2011; published online 10 February 2011)

\begin{abstract}
We present here an experimental study of the electrostatic coupling between the mechanical resonances of two nanowires or two nanotubes. This coupling occurs when the eigenfrequencies of the two resonators are matched by electrostatic tuning and it changes from a weak coupling to a strong coupling regime as the distance between the cantilevers is decreased. Linear coupling theory is shown to be in excellent agreement with the experimental data. (C) 2011 American Institute of Physics. [doi:10.1063/1.3553779]
\end{abstract}

Nanotubes and nanowires (NNs) are presently attracting considerable attention as resonators in nanoelectromechanical systems (NEMS). This is because their extremely small physical dimensions imply a high sensitivity of their resonance frequencies to external perturbations, thus opening new perspectives for both fundamental physics and technological applications. Respective examples are studying quantum mechanics on "macroscopical" objects, ${ }^{1,2}$ high resolution mass measurements ${ }^{3-5}$ and nanotelecommunications devices. ${ }^{6,7}$

The NNs' small dimensions also imply extremely small transduced signals and thus sophisticated device design and electronics for detection. This is a real bottleneck to the integration of NEMS devices. An interesting approach may be to increase signals by summing the responses of many NEMS resonating at the same eigenfrequencies and excited simultaneously. ${ }^{8}$ However two new aspects must be addressed before integration. First the fabrication of such frequency matched structures is still a great challenge and second, any coupling between resonators will lead to complex collective modes.

To investigate these two aspects we used "bottom up" fabricated NEMS structures of pairs of either multiwall carbon nanotubes (MWCNTs) or of silicon carbide nanowires (SiCNWs), resonating at close to the same eigenfrequencies. The eigenfrequencies of the NNs were matched by electrostatic induced strain ${ }^{9}$ which is a key element in this work. As described below, it is possible to match resonator pairs of specific dimensions. In short, lower frequency NEMS can have a stronger increase of the eigenfrequency with the applied voltage than higher frequency NEMS ${ }^{10}$ allowing the frequency matching. The coupling and collective modes of resonators must be taken into consideration before attempting device integration of several NNs that vibrate close to the same frequency. The coupling is usually either mechanical, via the substrate, or electrostatic, due to surface charges. The first mechanism was already studied showing that mechanical coupling is significant for "top down" fabricated resonators. ${ }^{8}$ The second mechanism was studied theoretically for carbon nanotubes arrays and important coupling was also found. ${ }^{11}$ In this letter we have studied experimentally the electrostatic coupling for "bottom up" fabricated nanoresonators for which the application of static voltages induces

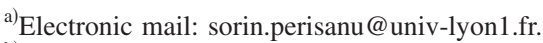

${ }^{b)}$ Electronic mail: pascal.vincent@univ-lyon1.fr.
}

large surface charges. A simple model of linear coupling is shown to be in excellent agreement with measured data.

Our first experiments were made on several MWCNTs glued on the same tungsten tip and placed inside a transmission electron microscope (TEM). A homemade support allows applying up to $2 \mathrm{kV}$ to the sample with respect to a spherical electrode mounted on a $z$ axis micrometric positioner. High frequency signals could be applied to either the tip, the spherical anode or a third electrode permitting gigahertz range excitation. ${ }^{12}$

Figure 1(a) presents a TEM image of two coupled MWCNTs vibrating together at the same eigenfrequency. Their Q-factor was $\simeq 1000$. There are two coupled modes for each applied voltage, with both resonators vibrating in both modes. The blue and red curves in Fig. 1(d) show the dependence on applied voltage of the eigenfrequencies of the two modes, designated as $f_{c j}(V)$ where c stands for coupled and $j \in\{H, L\}$ for the high or low frequency modes respectively. The nanotubes vibrated together as the $f_{c j}(V) \mathrm{s}$ were varied by over $20 \mathrm{MHz}$. The surface charges are the same sign which induces repulsive forces between cantilevers and there is no crossover between the two branches, a sure signature of coupled modes.

The two coupled MWCNTs were on the same mechanical support which could mean that the coupling was mechanical and not electrostatic. More detailed experiments were made inside a scanning electron microscope (SEM) on pairs of SiC nanowires individually mounted on two different etched tungsten tips, as shown in Figs. 1(b) and 1(c). A high dc voltage was applied on the first tip and a high frequency low voltage ac signal was brought to the second tip. The nanowires were respectively 40 and $53.4 \mu \mathrm{m}$ long and had diameters of 122 and $242 \mathrm{~nm}$, with intrinsic eigenfrequencies of 166 and $229 \mathrm{kHz}$. The Q-factors were $\approx 100$, but they can be increased by prior thermal treatment. ${ }^{13}$ Nanometric relative positioning of the two SiCNWs was achieved using an XYZ piezoelectrical nanomanipulator. This setup eliminates the mechanical coupling via the substrate, which proves that electrostatic interaction is sufficient to explain our results.

We have studied the dependence of the coupling on the distance $d$ between the SiCNWs ends, in the configuration of Fig. 1(c). The data in Fig. 1(e) corresponds to $d=6.5 \mu \mathrm{m}$, giving a strong coupling regime, where the minimal frequency separation between the two branches is still higher than the natural Lorentzian width given by their mechanical 

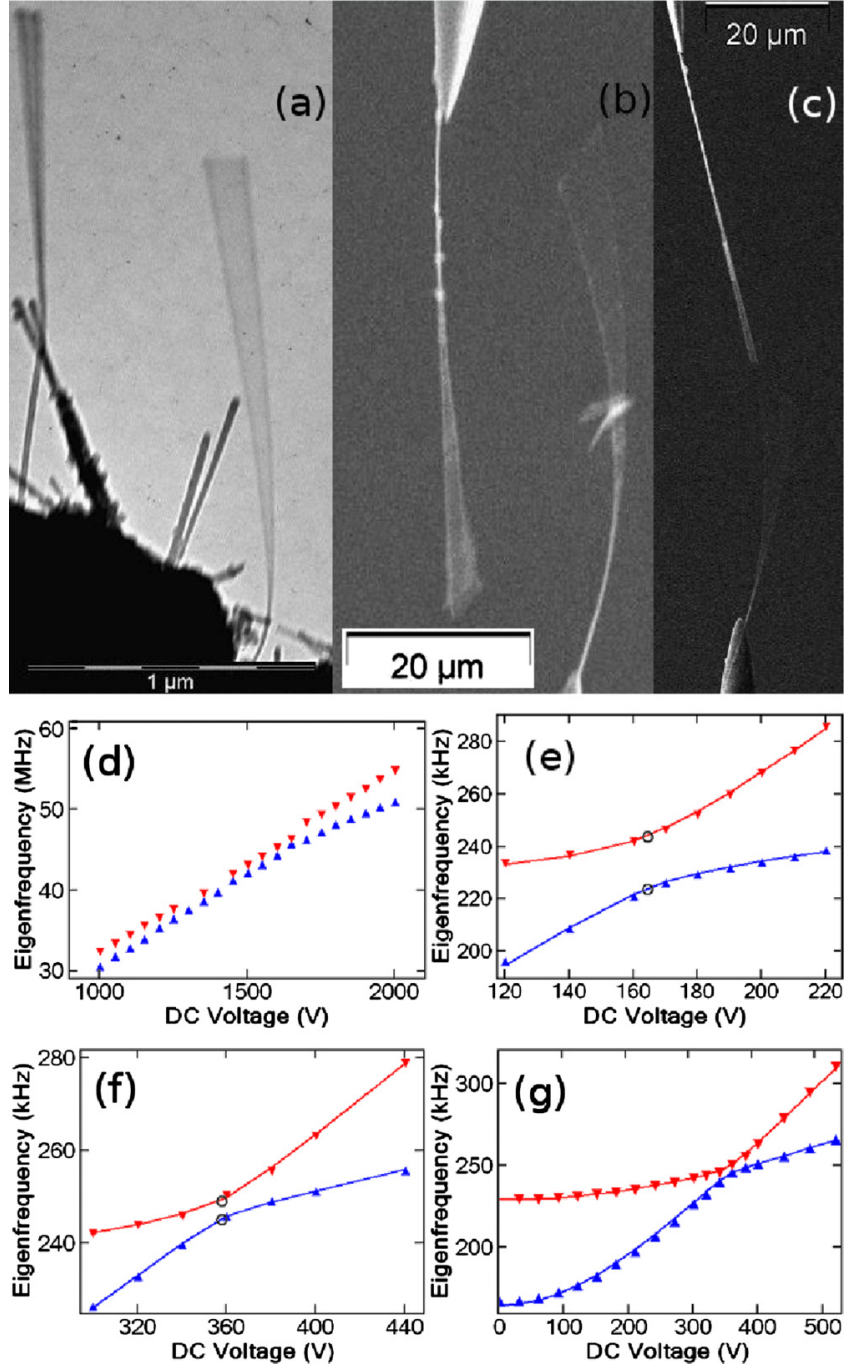

FIG. 1. (Color online) (a) TEM image of a MWCNT pair with coupled vibrations. [(b) and (c)] SEM images of a SiCNW pair undergoing coupled vibrations. The tungsten tips with the SiCNWs are glued on an XYZ piezoelectric manipulator allowing nanometric relative positioning. As we can see, coupling was observed for several geometrical configurations. The rest of this letter studies configurations similar to the one presented in (c) and differing just by the relative distance between the cantilever's apexes. (d) The crossover region in the $f_{c j}(V)$ curves for the pair of MWCNTs. [(e) and (f)] The crossover region in the $f_{c j}(V)$ curves for interapex distances of 6.5 and $42.4 \mu \mathrm{m}$ respectively. Fits with Eq. (2) (solid curves) give excellent agreement with experiment $(\boldsymbol{\Lambda}$ and $\boldsymbol{\nabla})$. (g) Experimental $f_{c j}(V)$ curves $(\boldsymbol{\Delta}$ and $\boldsymbol{\nabla}$ ) for the SiCNWs fitted with Eq. (2) (-) on a large voltage range.

Q-factor. For $d=42.6 \mu \mathrm{m}$ the coupling is no longer strong enough to clearly separate the two resonances. This behavior, presented in Figs. 1(f) and 1(g), corresponds to the weak coupling regime. Contrary to mechanical coupling, electrostatic coupling is present over a very wide frequency range due to the $V^{2}$ dependence of the coupling forces.

To achieve coupling the two cantilevers must be in close proximity and have nearly the same free resonance eigenfrequencies. The variation of the uncoupled eigenfrequency on electrostatic pulling has a universal dependence on voltage $f_{u}(V)$ with two free parameters: the zero voltage eigenfrequency, $f_{u}(0)$, and the characteristic tuning voltage, $V_{C}$, for which the elastic and electrostatic return forces are equal. ${ }^{10}$ Figure 2(a) shows that the dependence of the normalized frequency, $f_{u}(V) / f_{u}(0)$, on the normalized dc voltage, $V / V_{C}$, for six different MWCNTs follows the same curve within
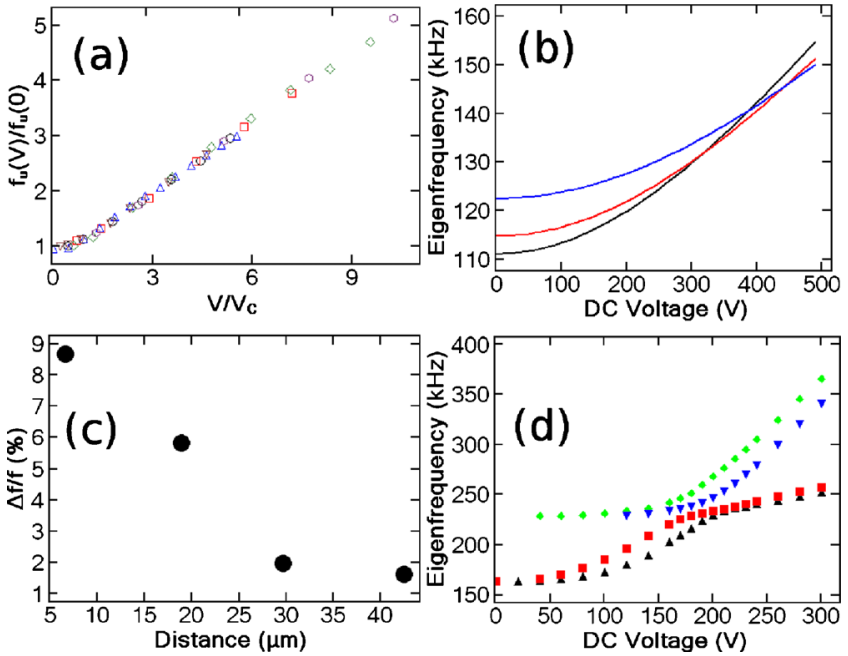

FIG. 2. (Color online) (a) Universal experimental tuning curve for six uncoupled MWCNTs under electrostatic stress. When the frequency $f_{u}(V)$ is normalized by the zero voltage frequency $f_{u}(0)$ and the dc voltage $V$ is normalized by the characteristic tuning voltage $V_{C}$, the curve $f_{u}(V) / f_{u}(0)$ as a function of $V / V_{C}$ is universal for all prism shaped resonators. (b) Calculated $f_{u}(V)$ curves for three uncoupled SiCNWs with lengths of 50, 55, and $64 \mu \mathrm{m}$ and radii of 20,25 , and $35 \mathrm{~nm}$. Those SiCNWs have close eigenfrequencies for voltages in the 300-500 V range. (c) Distance dependence of the coupling, measured as $\Delta f / f$ at the minimum separation between $f_{c H}(V)$ and $f_{c L}(V)$. The values for two small (large) distances correspond to a strong (weak) coupling regime. (d) $f_{c j}(V)$ coupling curves for the SiCNW pair with both polarizations. The low (high) frequency polarizations, represented with $\boldsymbol{\Delta}$ and $\boldsymbol{\nabla}(\boldsymbol{\square}$ and $\bullet$ ) couple independently, probably because they have similar eigendirections. Coupling was clearly observed in the 160-300 V range.

experimental error. Moreover, $f_{u}(0) \propto r / L^{2}$ and roughly $V_{C}$ $\propto r^{2} / L^{2}$ with $r$ and $L$ the NN's radius and length. ${ }^{10} \mathrm{~A}$ NN with a lower $f_{u}(0)$ can have a higher $f_{u}(0) / V_{C}$ than its higher $f_{u}(0) \mathrm{NN}$ partner. The higher $f_{u}(0) / V_{C}$ allows to tune the low frequency $\mathrm{NN}$ to the same (or higher) frequency at higher $\mathrm{V}$, thus allowing mode coupling.

Another aspect is that it is possible to show for a set of NNs with uniform distributions of $L$ and $r$ that there is a $2 / 3$ probability to achieve such a tuning between any NN pair. This probability increases to $100 \%$ if all the NNs have the same length. Furthermore, the calculations in Fig. 2(b) show that the differences in the eigenfrequencies in an $\mathrm{NN}$ forest can be strongly decreased by electrostatic tuning, thus increasing the coupling.

A detailed model of electrostatic coupling was developed in Ref. 11. We present here a simple linear model to explain our experimental data. Using $i, k \in\{1,2\}, i \neq k$, the differential system for the undriven and undamped motion is

$$
\ddot{X}_{i}+4 \pi^{2} f_{u i}^{2}(V) X_{i}=-4 \pi^{2} C_{i}(V)\left(X_{i}-X_{k}\right)
$$

with $X_{i}$ the apex displacements of the two cantilevers. $f_{u i}(V)$ are the NN's intrinsic eigenfrequencies given by the universal curve in Fig. 2(a). The "dots" represent time derivatives. $C_{i}(V)=C V^{2} / m_{i}(V)$ where $m_{i}(V)$ are the effective masses of the cantilevers as defined in Ref. 14. $C V^{2}$ is the linear coupling constant where the $V^{2}$ dependence is the general form for electrostatic forces. The eigenfrequencies of the coupled system are found by diagonalizing the system matrix 1: 


$$
\begin{aligned}
2 f_{c j}^{2}= & f_{u 1}^{2}(V)+f_{u 2}^{2}(V)+C_{1}(V)+C_{2}(V) \\
& \pm \sqrt{\left[f_{u 1}^{2}(V)-f_{u 2}^{2}(V)+C_{1}(V)-C_{2}(V)\right]^{2}+4 C_{1}(V) C_{2}(V)}
\end{aligned}
$$

where $+(-)$ corresponds to $f_{c H}\left(f_{c L}\right)$.

This expression contains five unknown parameters: the two zero voltage intrinsic eigenfrequencies $f_{u i}(0)$ and the two characteristic tuning voltages $V_{C i}$ describing the two universal $f_{u i}(V)$ dependencies shown in Fig. 2(a) for the uncoupled NNs, plus the coupling constant $C$, which depends only on the geometrical configuration since the $V^{2}$ dependence was taken account separately. Figures $1(\mathrm{e})-1(\mathrm{~g})$ show that this formula gives an excellent fit with experimental data, even over a large voltage range.

These fits to the experimental data determine precisely the minimum frequency separation $\Delta f \equiv \min \left[f_{c H}(V)\right.$ $\left.-f_{c L}(V)\right]$ and thus the strength of the coupling. Figure 2(c) shows $\Delta f$ normalized to the average eigenfrequency, $f$, at the corresponding voltage as a function of the distance between the wire apexes. We observed a decrease of $\Delta f / f$ by a factor of five as the separation was increased from 6.5 to $42.6 \mu \mathrm{m}$. This variation is compatible with coupling capacitances in the $a F$ range, but further electrostatic modeling is needed to extract an exact value.

Until now we have not introduced the fact that resonating NNs with circular crossections have two intrinsic perpendicular polarizations with nearly the same eigenfrequencies. ${ }^{14}$ In Figs. 1(e)-1(g) we have plotted the data for the coupled resonances of the two SiCNWs with the largest amplitudes as observed side on in the SEM. The data in Fig. 2(d) plots the $f_{c j}(V)$ curves for the two polarizations of both SiCNWs for which there is again the formation of a frequency gap. We have previously proved that the polarizations for an individual NN also couple to each other only by nonlinear terms ${ }^{14}$ which is distinct from these experiments which were limited to the linear regime. We therefore observed only couplings involving polarizations of different SiCNW, as presented in Fig. 1(c). A deeper study of NN polarizations coupling will be carried out in the near future.

In conclusion we have presented an experimental study of the electrostatic coupling between pairs of singly clamped MWCNTs or SiCNWs for which the eigenfrequencies were matched by differential electrostatic tuning. This coupling is very well explained by linear theory and the coupling constant strongly depends on the voltage difference and on the distance between the NNs. Experimental data on the coupling of several polarizations of the first eigenmode for a SiCNW pair was also presented. The strong electrostatic influence on the mechanics opens the perspective for coupled self oscillations in this configuration, as previously observed on individual NNs under Field Emission. ${ }^{15-17}$

This work is supported by the French National Research Agency (ANR) through the Nanoscience and Nanotechnology Program (Project NEXTNEMS No. ANR-07-NANO008-01 and Project NEMSPiezo No. ANR-08-P078-48-03).

${ }^{1}$ B. Lassagne, Y. Tarakanov, J. Kinaret, D. Garcia-Sanchez, and A. Bachtold, Science 325, 1107 (2009).

${ }^{2}$ G. A. Steele, A. K. Hüttel, B. Witkamp, M. Poot, H. B. Meerwaldt, L. P. Kouwenhoven, and H. S. J. van der Zant, Science 325, 1103 (2009).

${ }^{3}$ K. Jensen, K. Kim, and A. Zettl, Nat. Nanotechnol. 3, 533 (2008).

${ }^{4}$ B. Lassagne, D. Garcia-Sanchez, A. Aguasca, and A. Bachtold, Nano Lett. 8, 3735 (2008).

${ }^{5}$ H.-Y. Chiu, P. Hung, H. W. C. Postma, and M. Bockrath, Nano Lett. 8, 4342 (2008)

${ }^{6}$ K. Jensen, J. Weldon, H. Garcia, and A. Zettl, Nano Lett. 7, 3508 (2007).

${ }^{7}$ V. Gouttenoire, T. Barois, S. Perisanu, J. L. Leclercq, S. T. Purcell, P. Vincent, and A. Ayari, Small 6, 1060 (2010).

${ }^{8}$ R. B. Karabalin, M. C. Cross, and M. L. Roukes, Phys. Rev. B 79, 165309 (2009).

${ }^{9}$ S. T. Purcell, P. Vincent, C. Journet, and V. T. Binh, Phys. Rev. Lett. 89 276103 (2002).

${ }^{10}$ S. Perisanu, V. Gouttenoire, P. Vincent, A. Ayari, M. Choueib, M. Bechelany, D. Cornu, and S. T. Purcell, Phys. Rev. B 77, 165434 (2008).

${ }^{11}$ A. Isacsson and J. M. Kinaret, Phys. Rev. B 79, 165418 (2009).

${ }^{12}$ P. Poncharal, P. Vincent, J. M. Benoit, S. Perisanu, A. Ayari, M. Choueib, and S. T. Purcell, Nanotechnology 21, 215303 (2010).

${ }^{13}$ S. Perisanu, P. Vincent, A. Ayari, M. Choueib, M. Bechelany, D. Cornu, and S. T. Purcell, Appl. Phys. Lett. 90, 043113 (2007).

${ }^{14}$ S. Perisanu, T. Barois, A. Ayari, P. Poncharal, M. Choueib, S. T. Purcell, and P. Vincent, Phys. Rev. B 81, 165440 (2010).

${ }^{15}$ A. Ayari, P. Vincent, S. Perisanu, M. Choueib, V. Gouttenoire, M. Bechelany, D. Cornu, and S. T. Purcell, Nano Lett. 7, 2252 (2007).

${ }^{16}$ V. I. Kleshch, A. N. Obraztsov, and E. D. Obraztsova, JETP Lett. 90, 464 (2009).

${ }^{17}$ A. Lazarus, T. Barois, S. Perisanu, P. Poncharal, P. Manneville, E. de Langre, S. T. Purcell, P. Vincent, and A. Ayari, Appl. Phys. Lett. 96, 193114 (2010). 